\title{
Theoretical Study of Aminoacid-Based Ionic Liquids Interacting with Carbon Nanosystems
}

Cesar Herrera, ${ }^{a}$ Rafael Alcalde, ${ }^{a}$ Gregorio García, ${ }^{a}$ Mert Atilhan, ${ }^{\text {b and Santiago Aparicio }}{ }^{\text {a* }}$

${ }^{a}$ Department of Chemistry, University of Burgos, 09001 Burgos, Spain

${ }^{\mathrm{b}}$ Department of Chemical Engineering, Qatar University, P.O. Box 2713, Doha, Qatar

*Corresponding author: sapar@ubu.es

Supporting Information 
Table S1. Forcefield parameterization for compounds studied in this work.

The general form of the applied force field is:

$$
\begin{aligned}
E & =\sum_{\text {bonds }} k_{r}\left(r-r_{e q}\right)^{2}+\sum_{\text {angles }} k_{\theta}\left(\theta-\theta_{e q}\right)^{2}+E_{\text {tor }} \\
& +\sum_{i} \sum_{j}\left\{4 \varepsilon_{i j}\left[\left(\frac{\sigma_{i j}}{r_{i j}}\right)^{12}-\left(\frac{\sigma_{i j}}{r_{i j}}\right)^{6}\right]+\frac{q_{i} q_{j} e^{2}}{4 \pi \varepsilon_{0} r_{i j}}\right\}
\end{aligned}
$$

Dihedrals $\left(E_{t o r}\right)$ were described according to:

$$
E_{\text {tor }}=\sum_{\text {torsions }} k_{\phi}(1+\cos (m \phi-\delta))
$$

Improper dihedrals were described according to:

$$
E_{\text {improper }}=k_{\phi}\left(\phi-\phi_{0}\right)^{2}
$$




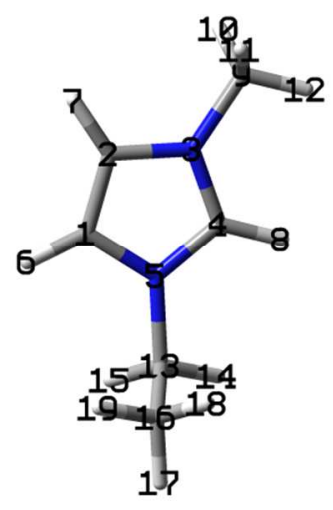

[EMIM] $^{+}$

\# Charges

\begin{tabular}{|c|c|}
\hline & $\begin{array}{c}\text { [EMIM] in } \\
\text { [EMIM] [GLY] }\end{array}$ \\
\hline \# & 9 \\
\hline 1 & -0.196205 \\
\hline 2 & -0.149642 \\
\hline 3 & 0.194066 \\
\hline 4 & -0.175725 \\
\hline 5 & 0.103379 \\
\hline 6 & 0.192142 \\
\hline 7 & 0.163767 \\
\hline 8 & 0.387847 \\
\hline 9 & -0.180845 \\
\hline 10 & 0.080037 \\
\hline 11 & 0.080857 \\
\hline 12 & 0.188487 \\
\hline 13 & 0.105395 \\
\hline 14 & 0.078680 \\
\hline 15 & 0.008925 \\
\hline 16 & -0.039745 \\
\hline 17 & 0.038870 \\
\hline 18 & 0.029136 \\
\hline 19 & 0.006011 \\
\hline & $\sum q$ \\
\hline total charge & 0.915437 \\
\hline
\end{tabular}

$\begin{array}{crcc}\text { \# Atoms } & & & \\ \# & \boldsymbol{\sigma}_{\boldsymbol{i i}} / \boldsymbol{\AA} & \boldsymbol{\varepsilon}_{\boldsymbol{i i}} / \mathbf{k J} \mathbf{~ m o l}^{\mathbf{- 1}} & \text { label } \\ 1 & 3.4000 & 0.359800 & \mathrm{C} 1 \\ 2 & 3.4000 & 0.359800 & \mathrm{C} 1 \\ 3 & 3.2500 & 0.711300 & \mathrm{~N} 1 \\ 4 & 3.4000 & 0.359800 & \mathrm{C} 2 \\ 5 & 3.2500 & 0.711300 & \mathrm{~N} 2 \\ 6 & 2.5110 & 0.062800 & \mathrm{H} 1 \\ 7 & 2.5110 & 0.062800 & \mathrm{H} 1 \\ 8 & 1.7820 & 0.062800 & \mathrm{H} 2 \\ 9 & 3.4000 & 0.457700 & \mathrm{C} 3 \\ 10 & 2.4710 & 0.065700 & \mathrm{H} 3 \\ 11 & 2.4710 & 0.065700 & \mathrm{H} 3 \\ 12 & 2.4710 & 0.065700 & \mathrm{H} 3 \\ 13 & 3.4000 & 0.457700 & \mathrm{C} 4 \\ 14 & 2.4710 & 0.065700 & \mathrm{H} 4 \\ 15 & 2.4710 & 0.065700 & \mathrm{H} 4\end{array}$




$\begin{array}{llll}16 & 3.4000 & 0.457700 & \text { C5 } \\ 17 & 2.6500 & 0.065700 & \text { H5 } \\ 18 & 2.6500 & 0.065700 & \text { H5 } \\ 19 & 2.6500 & 0.065700 & \text { H5 }\end{array}$

\# Bonds

$\begin{array}{crrr}\text { Atom } & \text { Numbers } & \boldsymbol{r}_{\boldsymbol{e q}} / \boldsymbol{\AA} & \boldsymbol{k}_{\boldsymbol{r}} / \mathbf{k J} \mathbf{~ m o l}^{\mathbf{- 1}} \AA^{-\mathbf{2}} \\ 1 & 2 & 1.3610 & 1715.4 \\ 1 & 5 & 1.3819 & 1673.6 \\ 1 & 6 & 1.0775 & 1527.2 \\ 2 & 3 & 1.3819 & 1673.6 \\ 2 & 7 & 1.0775 & 1527.2 \\ 3 & 4 & 1.3366 & 1673.6 \\ 3 & 9 & 1.4762 & 920.4 \\ 4 & 5 & 1.3366 & 1673.6 \\ 4 & 8 & 1.0779 & 1422.6 \\ 5 & 13 & 1.4762 & 920.4 \\ 9 & 10 & 1.0899 & 1292.8 \\ 9 & 11 & 1.0899 & 1292.8 \\ 9 & 12 & 1.0899 & 1292.8 \\ 13 & 14 & 1.0899 & 1292.8 \\ 13 & 15 & 1.0899 & 1292.8 \\ 13 & 16 & 1.5308 & 836.8 \\ 16 & 17 & 1.0935 & 1347.2 \\ 16 & 18 & 1.0935 & 1347.2 \\ 16 & 19 & 1.0935 & 1347.2\end{array}$

\# Angles

\begin{tabular}{rrrrl}
\multicolumn{1}{c}{ Atom Numbers } & $\boldsymbol{\theta}_{\boldsymbol{e q}} / \mathbf{d e g}$ & $\boldsymbol{k}_{\boldsymbol{\theta}} / \mathbf{k J} \mathbf{~ m o l}^{\mathbf{1}} \mathbf{r a d}^{\mathbf{2}}$ \\
2 & 1 & 5 & 107.28 & 543.92 \\
2 & 1 & 6 & 130.74 & 104.60 \\
5 & 1 & 6 & 122.04 & 104.60 \\
1 & 2 & 3 & 107.28 & 543.92 \\
1 & 2 & 7 & 130.74 & 104.60 \\
3 & 2 & 7 & 122.04 & 104.60 \\
2 & 3 & 4 & 108.25 & 543.92 \\
2 & 3 & 9 & 125.67 & 543.92 \\
4 & 3 & 9 & 125.75 & 543.92 \\
3 & 4 & 5 & 109.11 & 543.92 \\
3 & 4 & 8 & 125.44 & 104.60 \\
5 & 4 & 8 & 125.44 & 104.60 \\
1 & 5 & 4 & 108.25 & 543.92 \\
1 & 5 & 13 & 125.67 & 543.92 \\
4 & 5 & 13 & 125.75 & 543.92 \\
3 & 9 & 10 & 109.41 & 125.52 \\
3 & 9 & 11 & 109.41 & 125.52 \\
3 & 9 & 12 & 109.41 & 125.52 \\
10 & 9 & 11 & 108.44 & 148.54 \\
10 & 9 & 12 & 108.44 & 148.54 \\
11 & 9 & 12 & 108.44 & 148.54 \\
5 & 13 & 14 & 109.41 & 117.16 \\
5 & 13 & 15 & 109.41 & 117.16 \\
5 & 13 & 16 & 112.34 & 585.76 \\
14 & 13 & 15 & 108.44 & 148.54 \\
14 & 13 & 16 & 111.68 & 139.74 \\
15 & 13 & 16 & 111.68 & 139.74 \\
13 & 16 & 17 & 109.13 & 139.74 \\
13 & 16 & 18 & 109.13 & 139.74 \\
13 & 16 & 19 & 109.13 & 139.74 \\
17 & 16 & 18 & 107.24 & 148.54 \\
17 & 16 & 19 & 107.24 & 148.54 \\
18 & 16 & 19 & 107.24 & 148.54 \\
& & & & \\
3 & 3 & 102 &
\end{tabular}


Atom Numbers

$\delta / \operatorname{deg} \boldsymbol{k}_{\varphi} / \mathrm{kJ}^{\mathrm{mol}} \mathbf{m}^{-1}$

$180.0 \quad 58.575$

$180.0 \quad 12.551$

$180.0 \quad 15.551$

$180.0 \quad 8.368$

$180.0 \quad 58.575$

$0.0 \quad 0.000$

$180.0 \quad 12.551$

$180.0 \quad 0.000$

$180.0 \quad 58.575$

$0.0 \quad 0.000$

$180.0 \quad 12.551$

$180.0 \quad 0.000$

$180.0 \quad 58.593$

$180.0 \quad 12.551$

$180.0 \quad 0.000$

$180.0 \quad 0.000$

$0.0 \quad 0.000$

$0.0 \quad 0.000$

$0.0 \quad 0.000$

$180.0 \quad 0.823$

$180.0 \quad 0.823$

$180.0 \quad 0.823$

$180.0 \quad 58.575$

$180.0 \quad 0.000$

$180.0 \quad 12.551$

$180.0 \quad 0.000$

$0.0 \quad 0.000$

$0.0 \quad 0.000$

$0.0 \quad 0.840$

$180.0 \quad 0.823$

$180.0 \quad 0.823$

$180.0 \quad 0.420$

$0.0 \quad 0.000$

$0.0 \quad 0.000$

$0.0 \quad 0.000$

$0.0 \quad 0.823$

$0.0 \quad 0.823$

$0.0 \quad 0.823$

$0.0 \quad 0.823$

$0.0 \quad 0.823$

m

2

\# improper

Atom Numbers

$\begin{array}{ll}4 & 3 \\ 5 & 1 \\ 3 & 2 \\ 2 & 1 \\ 1 & 2\end{array}$

16

18

$\begin{array}{cc}\phi_{\boldsymbol{0}} / \mathbf{d e g} & \boldsymbol{k}_{\boldsymbol{\varphi}} / \mathbf{k J} \mathbf{~ m o l}^{-\mathbf{1}} \mathbf{r a d}^{\mathbf{2}} \\ 0.0 & 2.092 \\ 0.0 & 2.510 \\ 0.0 & 2.510 \\ 0.0 & 2.092 \\ 0.0 & 2.092\end{array}$




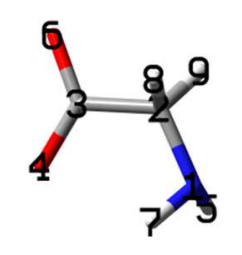

${ }^{[G L Y]^{-}}$

\# Charges
\begin{tabular}{|c|c|}
\hline & $\begin{array}{c}\text { [GLY] in } \\
{[\text { EMIM] [GLY] }}\end{array}$ \\
\hline$\#$ & q \\
\hline 1 & -1.0329 \\
\hline 2 & 0.4557 \\
\hline 3 & 0.7776 \\
\hline 4 & -0.8118 \\
\hline 5 & 0.3395 \\
\hline 6 & -0.9067 \\
\hline 7 & 0.3587 \\
\hline 8 & -0.0510 \\
\hline 9 & -0.0446 \\
\hline & $\sum 9$ \\
\hline total charge & -0.915437 \\
\hline
\end{tabular}

\# Atoms

$\begin{array}{cccc}\# & \boldsymbol{\sigma}_{\boldsymbol{i i}} / \boldsymbol{\AA} & \boldsymbol{\varepsilon}_{\boldsymbol{i i}} / \mathbf{~ k J ~ m o l}^{\mathbf{- 1}} & \text { label } \\ 1 & 3.2500 & 0.71128 & \mathrm{~N} 3 \\ 2 & 3.5000 & 0.45773 & \mathrm{C} 6 \\ 3 & 3.7500 & 0.35982 & \mathrm{C} 7 \\ 4 & 2.8600 & 0.97864 & \mathrm{O} 1 \\ 5 & 1.2000 & 0.06569 & \mathrm{H} 6 \\ 6 & 2.8600 & 0.97864 & \mathrm{O} 1 \\ 7 & 1.2000 & 0.06569 & \mathrm{H} 6 \\ 8 & 2.5000 & 0.06569 & \mathrm{H} 7 \\ 9 & 2.5000 & 0.06569 & \mathrm{H} 7\end{array}$

\# Bonds

\begin{tabular}{cccc}
\multicolumn{2}{c}{ Atom Numbers } & $\boldsymbol{r}_{\boldsymbol{e q}} / \boldsymbol{\AA}$ & $\boldsymbol{k}_{\boldsymbol{r}} / \mathbf{k J} \mathbf{~ m o l}^{\mathbf{- 1}} \AA^{\mathbf{- 2}}$ \\
1 & 2 & 1.449 & 1410.008 \\
1 & 5 & 1.010 & 1815.856 \\
1 & 7 & 1.010 & 1815.856 \\
2 & 3 & 1.522 & 1326.328 \\
2 & 8 & 1.090 & 1422.560 \\
2 & 9 & 1.090 & 1422.560 \\
3 & 6 & 1.229 & 2384.880 \\
3 & 4 & 1.229 & 2384.880
\end{tabular}

\# Angles

\begin{tabular}{|c|c|c|c|c|}
\hline \multicolumn{3}{|c|}{ Atom Numbers } & $\theta_{e q} / \operatorname{deg}$ & $k_{\theta} / \mathrm{kJ} \mathrm{mol}^{-1} \mathrm{rad}^{-2}$ \\
\hline 2 & 1 & 5 & 118.4 & 209.200 \\
\hline 2 & 1 & 7 & 118.4 & 209.200 \\
\hline 5 & 1 & 7 & 120.0 & 146.440 \\
\hline 1 & 2 & 3 & 110.1 & 263.592 \\
\hline 1 & 2 & 8 & 109.5 & 209.200 \\
\hline 1 & 2 & 9 & 109.5 & 209.200 \\
\hline 3 & 2 & 8 & 109.5 & 209.200 \\
\hline 3 & 2 & 9 & 109.5 & 209.200 \\
\hline 8 & 2 & 9 & 109.5 & 146.440 \\
\hline 2 & 3 & 6 & 120.4 & 334.720 \\
\hline
\end{tabular}




$\begin{array}{lllll}2 & 3 & 4 & 120.4 & 334.720 \\ 6 & 3 & 4 & 126.0 & 334.720\end{array}$

\# Dihedrals

Atom Numbers

$\begin{array}{llll}5 & 1 & 2 & 3 \\ 7 & 1 & 2 & 3 \\ 5 & 1 & 2 & 8 \\ 5 & 1 & 2 & 9 \\ 7 & 1 & 2 & 8 \\ 7 & 1 & 2 & 9 \\ 1 & 2 & 3 & 6 \\ 1 & 2 & 3 & 4 \\ 8 & 2 & 3 & 6 \\ 8 & 2 & 3 & 4 \\ 9 & 2 & 3 & 6 \\ 9 & 2 & 3 & 4\end{array}$

$\begin{array}{rcc}\boldsymbol{\delta} / \mathbf{d e g} & \boldsymbol{k}_{\boldsymbol{\varphi}} / \mathbf{k J ~ m o l}^{-\mathbf{1}} & \boldsymbol{m} \\ 0.00 & 3.000 & 3 \\ 0.00 & 3.000 & 3 \\ 0.00 & 15.000 & 3 \\ 0.00 & 15.000 & 3 \\ 0.00 & 15.000 & 3 \\ 0.00 & 15.000 & 3 \\ 180.00 & 30.000 & 2 \\ 180.00 & 30.000 & 2 \\ 180.00 & 15.000 & 2 \\ 180.00 & 15.000 & 2 \\ 180.00 & 15.000 & 2 \\ 180.00 & 15.000 & 2\end{array}$

